\title{
Therapeutic efficacy of cyclosporin A against spinal cord injury in rats with hyperglycemia
}

\author{
ZHI-RONG CHEN ${ }^{1}$, YI MA ${ }^{2}$, HAO-HUI GUO ${ }^{1}$, ZHI-DONG LU ${ }^{1}$ and QUN-HUA JIN ${ }^{1}$ \\ ${ }^{1}$ Department of Orthopedics, General Hospital of Ningxia Medical University; ${ }^{2}$ Department of Pathology and Physiology, \\ Ningxia Medical University, Yinchuan, Ningxia 750004, P.R. China
}

Received May 20, 2016; Accepted July 24, 2017

DOI: $10.3892 / \mathrm{mmr} .2018 .8422$

\begin{abstract}
The present study aimed to explore the therapeutic effects of cyclosporin A (CsA) on spinal cord injury (SCI) in rats with hyperglycemia and to identify a novel potential method to treat SCI in the presence of hyperglycemia. Female Sprague-Dawley (SD) rats were randomly allocated into four groups: Sham, SCI, SCI+hyperglycemia and SCI+hyperglycemia+CsA groups. Streptozotocin-induced hyperglycemic SD rats and a weight-drop contusion SCI model were established. The Basso, Beattie, Bresnahan scale and inclined plane test were used to evaluate the neurological function of the rats. Flow cytometric assay was performed to detect the apoptotic rates of cells in the spinal cord. ELISA and western blot analysis were performed to determine the levels of interleukin (IL)-10, tumor necrosis factor (TNF)- $\alpha$, cyclophilin-D (Cyp-D) and apoptosis-inducing factor (AIF). The results demonstrated that CsA significantly improved the neurological function of the SCI rats with hyperglycemia. CsA markedly reduced the number of apoptotic cells exaggerated by hyperglycemia in the spinal cord of the SCI rats. CsA significantly decreased the expression levels of IL-10, TNF- $\alpha$, Cyp-D and AIF in the spinal cord of the SCI rats. Overall, the present study revealed a significant role of CsA in the treatment of SCI in the presence of hyperglycemia by inhibiting the apoptosis of spinal cord cells.
\end{abstract}

\section{Introduction}

Spinal cord injury (SCI) is a serious neurological and pathological disease, which directly damages spinal cord parenchyma and the associated spinal nerves. There are $\sim 12,000$ new cases of SCI annually in the USA (1), the majority of which are caused by vehicle accidents, falls or violence (2). Secondary complications,

Correspondence to: Dr Qun-Hua Jin, Department of Orthopedics, General Hospital of Ningxia Medical University, 1160 Shengli Street, Yinchuan, Ningxia 750004, P.R. China

E-mail: jqhgsysu@163.com

Key words: spinal cord injury, hyperglycemia, cyclosporin A, neurological function, apoptosis, inflammation which occur in the endocrinologic, cardiovascular, gastrointestinal, musculoskeletal and respiratory systems, are common in cases of SCI (3), which often lead to high morbidity and mortality rates. At present, the primary therapeutic methods for SCI include drugs (4), hypothermia therapy (5), stem cell transplantation (2) and immunotherapy (6). Despite considerable improvement in therapeutic methods of SCI in previous decades, the long-term outcomes of patients with SCI remain poor. Thus, it is important to identify and implement novel and more effective therapeutic strategies for patients with SCI.

Hyperglycemia, often caused by diabetes mellitus or non-diabetic hyperglycemia, is considered to be a potentially life-threatening clinical condition. Food diet, genetic factors, obesity and drugs may induce hyperglycemia. Increasing evidence has shown that hyperglycemia can impair several systems, including the cardiovascular system (7), auditory system (8), urinary system (9) and visual system (10). Meng et al (11) revealed that hyperglycemia can lead to neuroinflammation by activating the NLR family pyrin domain containing 1 inflammasome, which may be a mechanism of diabetes-associated neural injury in diabetic rats. Dai et al (12) demonstrated that neuropeptide FF-amide peptide precursor (NPFF) contributed to diabetic nerve injury recovery through NPFF receptor 2, and neuropeptide FF was a potential neuroregenerative factor for nerve injury associated with hyperglycemia. Hyperglycemia is an important indicator of health in patients with SCI (13); however, to date, the underlying association between hyperglycemia and SCI remains to be fully elucidated.

To our knowledge, there is no consensus on the treatment of patients with SCI with hyperglycemia. In previous years, studies have showed that mitochondria are important in nerve cell death following contusive SCI, which primarily regulate cell apoptosis through the mitochondrial permeability transition pore (mPTP), a non-specific giant channel (14). Therefore, inhibiting mPTP channels can prevent and reduce cell apoptosis. Cyclosporin A (CsA), an 11 amino acid cyclic peptide separated from Tolypocladium inflatumin culture medium, is regarded as an immunosuppressant. CsA can inhibit T lymphocytes through the following two pathways: i) CsA inhibits the generation of interleukin (IL)-2 (15); ii) CsA inhibits generation of the IL-2 receptor (16). CsA has been widely used in the treatment of various diseases, including aplastic anemia (17), nephritic syndrome (18), rheumatoid 
arthritis (19) and psoriasis (20). Studies have shown that CsA inhibits cyclophilin-D (Cyp-D), and then prevents the formation of mPTP (21). CsA can effectively reduce cerebral ischemic injury aggravated by hyperglycemia (22). However, to date, whether CsA can also reduce the severity of SCI aggravated by hyperglycemia remains to be elucidated.

The present study aimed to focus on the cell apoptotic pathway associated with mitochondria in the spinal cord, which may be used to clarify the underlying mechanism of CsA in the treatment of SCI with hyperglycemia. The results may provide a potential therapeutic drug and effective strategy for the treatment of patients with SCI and hyperglycemia.

\section{Materials and methods}

Animals and groups. A total of 40 adult female healthy SPF Sprague-Dawley rats, weighing 220-240 g, were obtained from Shanghai Laboratory Animal Center Co., Ltd. (Shanghai, China). Animal experimental trials were approved by the Animal Use and Ethics Committee of Ningxia Medical University (Yinchuan, China). The 40 rats were randomly allocated into four groups: Sham group, $(n=10)$, in which rats underwent laminectomy only; SCI group $(\mathrm{n}=10)$, in which rats underwent SCI only; SCI+hyperglycemia group $(n=10)$, in which rats received streptozotocin (STZ) prior to SCI; and $\mathrm{SCI}+$ hyperglycemia+CsA group $(\mathrm{n}=10)$, in which rats received STZ prior to SCI, and CsA following SCI.

All animals were allowed to adapt to the environment for 3 weeks prior to the trial. The rats of the SCI+hyperglycemia group and $\mathrm{SCI}+$ hyperglycemia+CsA group were intraperitoneally injected with STZ (Sigma-Aldrich; Merck Millipore, Darmstadt, Germany) at $30 \mathrm{mg} / \mathrm{kg}$ body weight dissolved in citrate buffer ( $\mathrm{pH} 4.5)$ every day to induce hyperglycemia. The rats were considered to have hyperglycemia if their blood glucose levels were maintained at $>16.8 \mathrm{mmol} / 148 \mathrm{~h}$ following STZ injection. The rats were maintained in ambient temperature $\left(20-25^{\circ} \mathrm{C}\right)$ with a $12: 12 \mathrm{dark} /$ light cycle and were provided with free access to food and ultrapure water. All attempts were made to reduce the number of animals used in the present study and to minimize their suffering.

Spinal cord injury modeling. The rats were anesthetized with $1.25 \%$ halothane in an oxygen/nitrous oxide $(30 / 70 \%)$ gas mixture. The dorsal skin was incised and the soft tissue was dissected, following which a laminectomy was performed carefully at the T10 vertebrae, and the vertebral column was completely exposed (Fig. 1). Contusion injury was performed in accordance with Allen's method (23). A weight of $10 \mathrm{~g}$ was dropped from a height of $5 \mathrm{~cm}$ onto the exposed spinal cord, and the impounder remained for $20 \mathrm{sec}$ following withdrawal to produce a moderate contusion. Bacitracin ointment (Qualitest Pharmaceuticals, Huntsville, AL, USA) was used to avoid infections, and another $5 \mathrm{ml}$ of normal saline solution was injected subcutaneously. The animals were allowed to recover on a water-circulating heating pad at $37^{\circ} \mathrm{C}$ (Gaymar Industries, Orchard Park, NY, USA). The animals in the Sham group received only a laminectomy for $1 \mathrm{~min}$. Following contusive SCI, twice daily bladder expression was performed until bladder function had recovered. Two of the hyperglycemia rats died within the first week following contusive SCI.
The SCI+hyperglycemia+CsA group rats were subcutaneously injected with CsA (4 mg/kg body weight; Novartis, Basal, Switzerland) every day. According to the trial, the rats were sacrificed using an overdose of pentobarbital sodium on day 7 , with the exception of 18 rats, which were used to assess locomotion recovery.

Neurological function assessment. The Basso, Beattie, Bresnahan (BBB) locomotor rating scale (24) was used to detect the recovery of motor function following contusive SCI. A score of 0 was considered to indicate no spontaneous mobility, whereas a score of 21 was considered to indicate complete mobility. The ability of an animal to maintain postural stability was assessed using Rivlin's inclined plane test (25). The maximum angle at which the rats were able to maintain a constant position on an oblique board for at least $5 \mathrm{sec}$ was considered the critical value and was recorded. Two independent examiners who were blinded to treatment observed the rats prior to injury, and at 1, 3, 7, 14 and 21 days post-injury.

Flow cytometric analysis of cell apoptosis. Several $5-\mu \mathrm{m}$-thick spinal cord sections containing the contusion sites were isolated. A single-cell suspension was obtained by trypsinization, and the rate of apoptosis was measured using an Annexin V Apoptosis Detection kit according the manufacturer's protocol. Finally, the apoptotic cells were examined and quantified using flow cytometry (BD Biosciences, San Diego, CA, USA).

ELISA analysis. Total proteins from the spinal cord tissues were extracted in RIPA lysis buffer (Beyotime, Haimen, China) containing protease inhibitor phenylmethanesulfonyl fluoride (Beyotime). A BCA protein assay kit (Thermo Fisher Scientific, Inc., Waltham, MA, USA) was used to assess protein concentrations. The concentrations of interleukin-10 (IL-10) and tumor necrosis factor- $\alpha$ (TNF- $\alpha$ ) were measured using respective ELISA kits according to the manufacturer's protocol, and analyzed using a microplate reader (Dynex Technologies, Chantilly, CA, USA). The values are expressed as $\mathrm{pg} / \mathrm{ml}$.

Western blot analysis. To determine the relative protein expression levels of Cyp-D and apoptosis-inducing factor (AIF), equal amounts of protein $(40 \mu \mathrm{g})$ from each group were separated by SDS-PAGE $(12 \%$ gel) and then transferred onto a PVDF membrane (Millipore, Billerica, MA, USA). Targeted proteins were detected by incubation overnight at $4^{\circ} \mathrm{C}$ with primary antibodies against Cyp-D (dilution, 1:500; cat. no. PA5-31061; Invitrogen; Thermo Fisher Scientific, Inc.) and AIF (dilution, 1:200; cat. no. 5318; Cell Signaling Technology, Inc., Danvers, MA, USA), followed by incubation for $1 \mathrm{~h}$ at $37^{\circ} \mathrm{C}$ with horseradish peroxidase-conjugated secondary antibody (dilution, 1:500; cat. no. 29-0382-76; GE Healthcare, Chicago, IL, USA). Signals were visualized using ECL Advance reagent (Beyotime Institute of Biotechnology) and quantified using ImageJ 2.0 software (National Institutes of Health, Bethesda, MD, USA). The expression levels of target protein were determined following normalization to individual $\beta$-actin levels. 
Table I. Body weights and blood glucose levels of rats.

\begin{tabular}{lcc}
\hline Group & $\begin{array}{c}\text { Body } \\
\text { weight } \\
(\mathrm{g})\end{array}$ & $\begin{array}{c}\text { Blood } \\
\text { glucose } \\
(\mathrm{mmol} / \mathrm{l})\end{array}$ \\
\hline Pre-STZ injection & & \\
Sham & $233 \pm 6$ & $4.2 \pm 0.5$ \\
SCI & $232 \pm 6$ & $4.1 \pm 0.5$ \\
SCI+hyperglycemia & $231 \pm 6$ & $4.2 \pm 0.6$ \\
SCI+hyperglycemia+CsA & $233 \pm 6$ & $4.3 \pm 0.6$ \\
4 weeks post-STZ injection/pre-SCI & & \\
Sham & $233 \pm 6$ & $4.2 \pm 0.6$ \\
SCI & $232 \pm 6$ & $4.2 \pm 0.5$ \\
SCI+hyperglycemia & $209 \pm 15^{\mathrm{a}}$ & $24.3 \pm 4.9^{\mathrm{a}}$ \\
SCI+hyperglycemia+CsA & $211 \pm 16^{\mathrm{b}}$ & $23.8 \pm 5.0^{\mathrm{a}}$ \\
\hline
\end{tabular}

${ }^{\mathrm{a}} \mathrm{P}<0.001 ;{ }^{\mathrm{b}} \mathrm{P}<0.01$. SCI, spinal cord injury; CsA, cyclosporin A; STZ, streptozotocin.

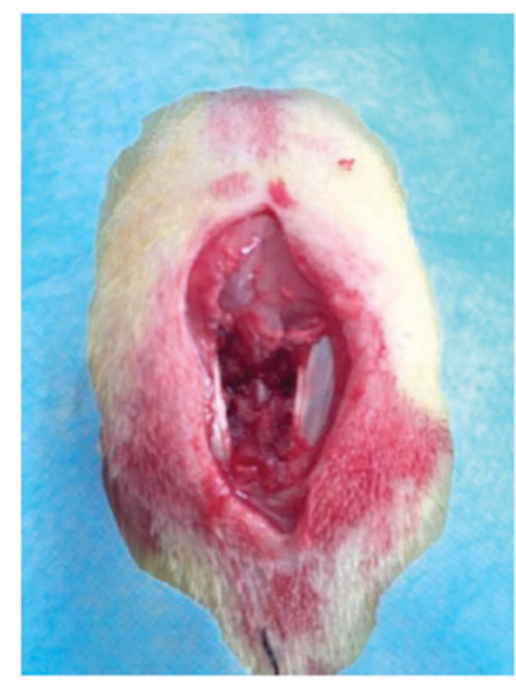

Figure 1. Exposed T10 vertebral column of a rat.

Statistical analysis. All statistical analyses were performed using SPSS 19.0 (IBM SPSS, Armonk, NY, USA) and GraphPad Prism 5.0 software (GraphPad Software, Inc., La Jolla, CA, USA). All data are presented as the mean \pm standard deviation. Unpaired Student's t-test was used to compare the experimental groups. $\mathrm{P}<0.05$ was considered to indicate a statistically significant difference.

\section{Results}

Body weight and blood glucose. As shown in Table I, prior to STZ injection, no significant differences in weight or blood glucose were observed among the four groups of rats. At 4 weeks post-STZ injection, prior to SCI, the, rats of the SCI+hyperglycemia group and $\mathrm{SCI}+$ hyperglycemia+CsA group had significantly lower body weights and higher blood glucose levels $(\mathrm{P}<0.05)$, compared with the rats of the Sham group and $\mathrm{SCI}$ group, indicating that the rats exhibited hyperglycemia.
A

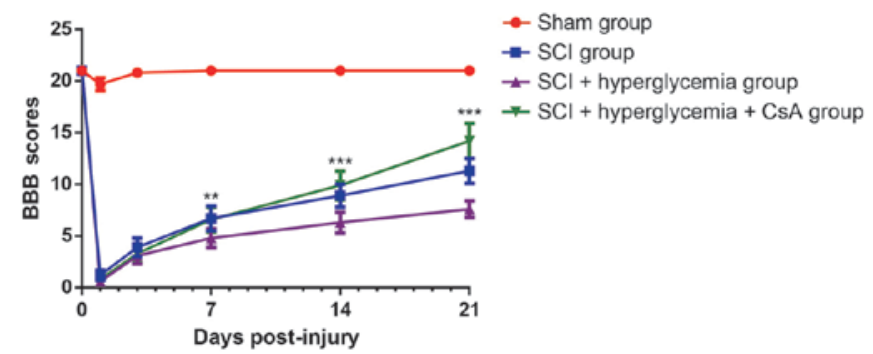

B

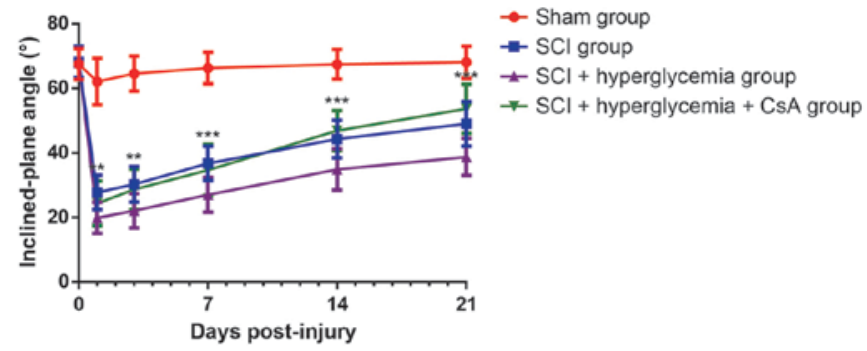

Figure 2. Results of the motor function assessment of the rats. (A) BBB scores (B) Inclined-plate angles. ${ }^{* *} \mathrm{P}<0.01,{ }^{* * *} \mathrm{P}<0.001 \mathrm{SCI}+$ hyperglycemia+CsA group vs. SCI+hyperglycemia group. SCI, spinal cord injury; CsA, cyclosporin A; BBB, Basso, Beattie, Bresnahan.

Neurological function assessment. As shown in Fig. 2A, the BBB scores of the rats in Sham group were similar to the normal values at each time point. Compared with the rats in the Sham group, the rats with SCI showed lower BBB scores at each time point, and the differences were statistically significant $(\mathrm{P}<0.001)$, indicating severe impairment of neurological function. The BBB scores of the SCI rats with hyperglycemia at 21 days remained $<10$, whereas the rats treated with CsA had scores above 10 points, and the differences were statistically significant $(\mathrm{P}<0.001)$. As shown in Fig. 2B, following contusive SCI, the inclined-plate angles of the rats were decreased, compared with those in the Sham group at each time point, and the differences were statistically significant $(\mathrm{P}<0.001)$. The inclined-plate angles of the rats treated with CsA increased to significantly higher angles, compared with those of rats in the SCI+hyperglycemia group at 14 and 21 days post-injury $(\mathrm{P}<0.001)$. These results suggested that hyperglycemia exaggerated the neurological function of the rats following contusive SCI, and that CsA accelerated the recovery of neurological function.

Flow cytometric analysis of apoptotic cells. As shown in Fig. 3, in the Sham group, almost no apoptotic cells were observed. However, the numbers of apoptotic cells in the spinal cord were significantly increased following contusive SCI $(\mathrm{P}<0.001)$, indicating increased apoptotic activity of cells in the spinal cords. Additionally, the number of apoptotic cells in the SCI+hyperglycemia group was higher, compared with that in the SCI group $(\mathrm{P}=0.002)$, and the number of apoptotic cells in the SCI+hyperglycemia+CsA group was significantly lower, compared with that of the SCI+hyperglycemia group $(\mathrm{P}<0.001)$. These results suggested that hyperglycemia exaggerated the apoptosis of spinal cord cells following contusive SCI, and that CsA inhibited this apoptosis. 

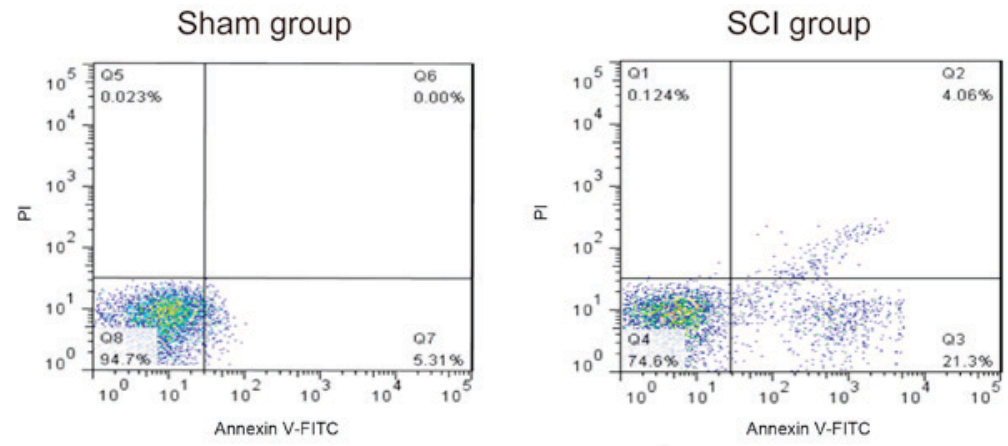

$\mathrm{SCl}+$ hyperglycemia group

$\mathrm{SCl}+$ hyperglycemia $+\mathrm{CsA}$ group
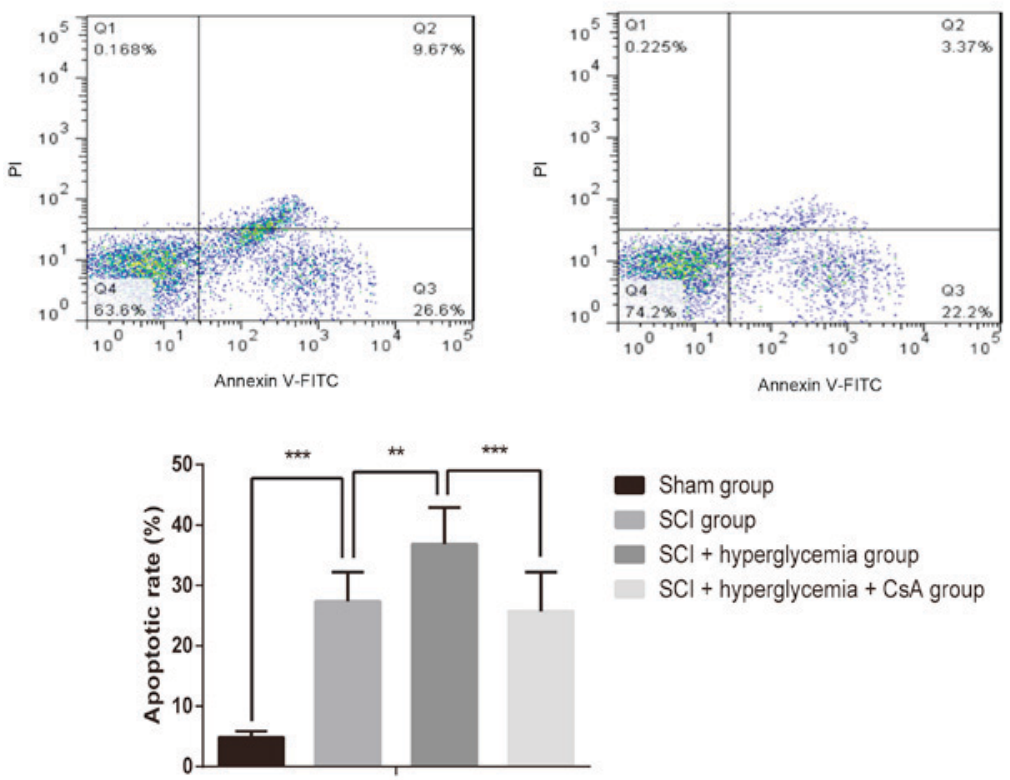

Figure 3. Flow cytometry assays to detect apoptotic cells in the spinal cord. ${ }^{* *} \mathrm{P}<0.01,{ }^{* * *} \mathrm{P}<0.001$. SCI, spinal cord injury; CsA, cyclosporin $\mathrm{A}$.
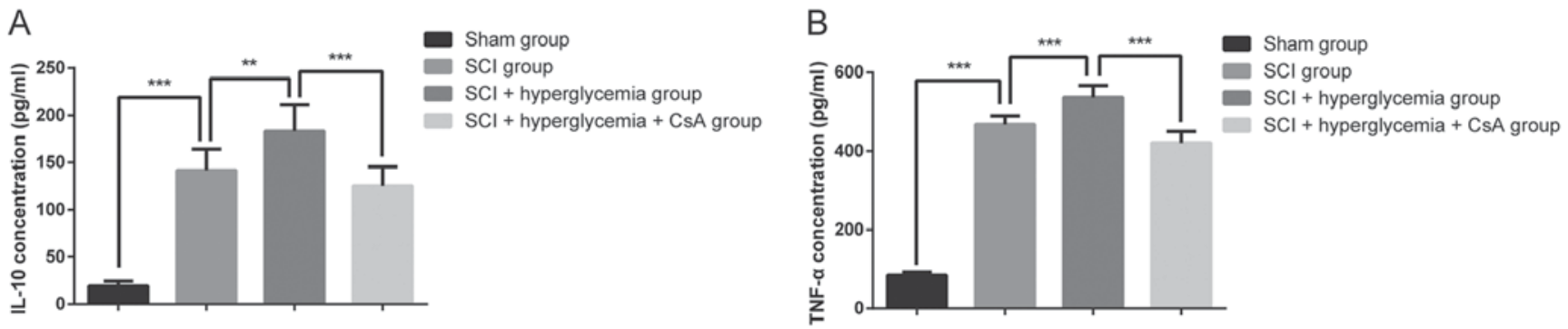

Figure 4. ELISA analysis of expression levels of IL-10 and TNF- $\alpha$ in the spinal cord. (A) Expression levels of IL-10. (B) Expression levels of TNF- $\alpha .^{* *} \mathrm{P}<0.01$, ${ }^{* * * *} \mathrm{P}<0.001$. SCI, spinal cord injury; CsA, cyclosporin A; IL-10, interleukin-10; TNF- $\alpha$, tumor necrosis factor- $\alpha$.

ELISA analysis of IL-10 and TNF- $\alpha$. As shown in Fig. 4A and $\mathrm{B}$, according to the results of ELISA analysis, the rats with SCI exhibited a significant increase in the expression levels of IL-10 and TNF- $\alpha$, compared with those in the Sham group $(\mathrm{P}<0.001)$. In addition, the expression levels of IL-10 and TNF- $\alpha$ in the rats in the SCI+hyperglycemia group were higher, compared with those in the rats in the SCI group $(\mathrm{P}=0.003$ and $\mathrm{P}<0.001$, respectively), whereas the expression levels of IL-10 and TNF- $\alpha$ in the rats in the SCI+hyperglycemia+CsA group were significantly lower, compared with those in the SCI+hyperglycemia group $(\mathrm{P}<0.001)$. These results suggested that hyperglycemia exaggerated the inflammation in the spinal cord following contusive SCI, and that CsA alleviated the inflammation in the spinal cord.

Western blot analysis of the protein expression of Cyp-D and AIF. As shown in Fig. 5, according to the results of the western blot analysis, compared with the rats of the Sham group, the rats with SCI exhibited increased protein expression levels of Cyp-D and AIF in the spinal cord $(\mathrm{P}<0.001)$. The protein levels of Cyp-D and AIF in the spinal cords of rats in the SCI+hyperglycemia group were significantly higher, compared with those of rats in the SCI group $(\mathrm{P}=0.026$ and $\mathrm{P}=0.001$, respectively), whereas the protein expression 


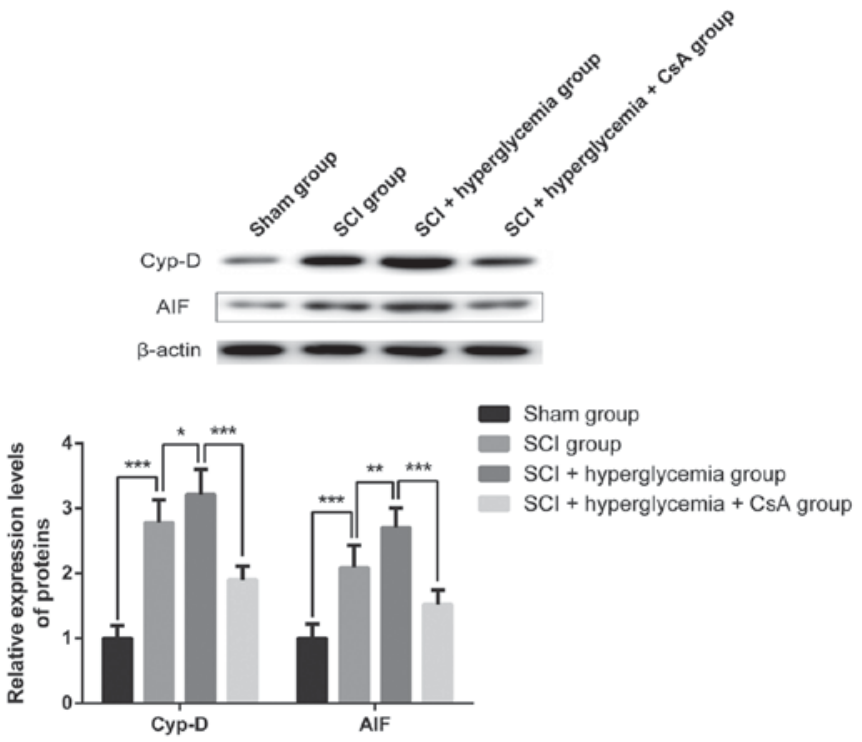

Figure 5. Western blot analysis of protein expression levels of Cyp-D and AIF in the spinal cord. ${ }^{*} \mathrm{P}<0.05,{ }^{* *} \mathrm{P}<0.01,{ }^{* * * *} \mathrm{P}<0.001$. SCI, spinal cord injury CsA, cyclosporin A; Cyp-D, cyclophilin-D; AIF, apoptosis-inducing factor.

levels of Cyp-D and AIF in the spinal cords of rats in the SCI+hyperglycemia+CsA group were lower, compared with those in the $\mathrm{SCI}+$ hyperglycemia group $(\mathrm{P}<0.001)$. These results indicated that hyperglycemia exaggerated the impairment of mitochondrial function in the spinal cord cells following contusive SCI, and that CsA inhibited the impairment of mitochondrial function.

\section{Discussion}

The pathogenesis of SCI can usually be divided into two stages, primary injury and secondary injury (26). Through the establishment of SCI rat models, the present study aimed to reveal the therapeutic effects of CsA on SCI of rats with hyperglycemia and to identify a novel method to treat SCI with hyperglycemia. The results showed that hyperglycemia aggravated the secondary injury of SCI, whereas that CsA improved the motor capacity of rats and reduced inflammation in the spinal cord following contusive SCI. This indicated that CsA effectively alleviated SCI impairment aggravated by hyperglycemia.

As is already known, the main clinical manifestations of SCI are motor and sensory disorders with a series of serious complications $(27,28)$. The BBB locomotor rating scale and Rivlin's inclined plane test are commonly used methods, which can evaluate changes in the motor capacity of experimental animals. In the present study, the results showed that the $\mathrm{BBB}$ scores and inclined-plate angles were decreased in the SCI rats. In addition, the BBB scores and inclined-plate angles were significantly decreased in the SCI rats with hyperglycemia, whereas CsA partially improved the decreased BBB scores and inclined-plate angles. These results indicated that the SCI rat models were established successfully, and that CsA improved the neurological function of the SCI rats with hyperglycemia.

Previous studies have found that inflammatory cytokines and inflammatory response in the spinal cord are closely correlated with the pathogenesis of SCI (29). For example, interleukin-4 (IL-4) is an anti-inflammatory cytokine, which is crucial in regulating acute macrophage activation and confining secondary cavity formation following contusive SCI (30). Fenn et al (31) showed that IL-4 reprogrammed active microglia to a phenotype promoting improved neurite growth, which was impaired with age, associated with reduced functional recovery following contusive SCI. IL-10 is also an anti-inflammatory cytokine, which can reduce pain-associated behaviors following contusive SCI (32). In addition, Lin et al (33) demonstrated that downregulation of IL-11 significantly decreased the expression of TNF- $\alpha$, and had therapeutic benefits by alleviating the inflammatory response and improving the recovery of SCI. This indicated that inhibiting TNF- $\alpha$ may contribute to recovery from SCI. To date, whether hyperglycemia can aggravate the severity of SCI remains unclear. In the present study, the expression levels of IL-10 and TNF- $\alpha$ were significantly higher in SCI rats with hyperglycemia. These results demonstrated that hyperglycemia aggravated the severity and inflammatory response of SCI. At present, there are no effective therapies for SCI due to the frequent occurrence of secondary injury, including edema, ischemia, excitotoxicity, inflammation, oxidative damage and apoptotic cell death (34).

Mitochondria, a primary structure responsible for generating ATP, are important in maintaining the normal function and growth of eukaryotic cells. When mitochondrial damage occurs, the cells lack energy supply due to the inability to produce sufficient ATP, which leads to cell dysfunction and even necrosis. Mitochondrial membrane perturbations are also involved in calcium overloading, the opening of mPTP channels and the release of apoptotic mediators into the cytoplasm (35). mPTP channels consist predominantly of three proteins, Cyp-D, adenine nucleotide translocator 1 and the voltage-dependent anion channel. mPTP channels are induced to open by pro-apoptotic stimuli, which cause loss of mitochondrial integrity and allow the release of several molecules into the cytoplasm (36). Mitochondria function as $\mathrm{Ca}^{2+}$ 'sinks', taking up excess $\mathrm{Ca}^{2+}$ to maintain $\mathrm{Ca}^{2+}$ levels in the cytosol, and mitochondrial function is severely impaired following contusive SCI (34). AIF is also a mitochondrial protein, which can exert a pro-apoptotic effect. It is released from mitochondria and translocates to nuclei, followed by the induction of chromatin condensation and DNA degradation during cell apoptosis (37). To date, previous studies have indicated that CsA has a therapeutic effect on a number of diseases through various pathways, including reducing the proportion of CD4(+) T cells and the expression level of interferon-i for the treatment of aplastic anemia (38). Guan et al (39) demonstrated that CsA has a protective effect in improving mitochondrial function through inhibiting mitofusin proteins, which are located on the outside of mitochondrial membranes and regulate mitochondrial fusion. In the present study, the number of apoptotic cells, and the protein levels of Cyp-D and AIF in the spinal cord were higher in the SCI rats with hyperglycemia, indicating that hyperglycemia accelerated the apoptosis of spinal cord cells following contusive SCI. CsA reduced the apoptosis of spinal cord cells and increased the protein expression levels of Cyp-D and AIF. Consistently, Kim et al (40) showed that CsA-mediated Cyp-D inhibition, which protected retinal 
ganglion cells against mitochondrial dysfunction, providing therapeutic potential for ischemic injury. These results demonstrated that CsA effectively reduced the SCI impairment aggravated by hyperglycemia.

In conclusion, the results of the present study showed that hyperglycemia aggravated the severity of SCI, and that CsA effectively reduce the SCI aggravated by hyperglycemia. These results provide a novel and relatively more effective potential therapeutic strategy for patients with SCI and hyperglycemia. However, the present study had several limitations, including lacking of in vitro experiments and no determination of the expression levels of IL-10 and TNF- $\alpha$ at each time point. Future investigations aim to examine whether CsA has a relative effect on spinal cord nerve cells in vitro and determine its specific mechanisms.

\section{Acknowledgements}

This study was funded by the Natural Science Foundation of China (grant no. 81360196) and the Natural Science Foundation of Ningxia (grant no. NZ15157).

\section{References}

1. Saunders LL, Clarke A, Tate DG, Forchheimer M and Krause JS: Lifetime prevalence of chronic health conditions among persons with spinal cord injury. Arch Phys Med Rehabil 96: 673-679, 2015.

2. Falnikar A, Li K and Lepore AC: Therapeutically targeting astrocytes with stem and progenitor cell transplantation following traumatic spinal cord injury. Brain Res 1619: 91-103, 2015.

3. Gorman PH: The review of systems in spinal cord injury and dysfunction. Continuum (Minneap Minn) 17: 630-634, 2011.

4. Kabu S, Gao Y, Kwon BK and Labhasetwar V: Drug delivery, cell-based therapies, and tissue engineering approaches for spinal cord injury. J Control Release 219: 141-154, 2015.

5. Wang $\mathrm{J}$ and Pearse DD: Therapeutic hypothermia in spinal cord injury: The status of its use and open questions. Int J Mol Sci 16 : 16848-16879, 2015.

6. Wang YT,Lu XM, Chen KT, Shu YH and Qiu CH: Immunotherapy strategies for spinal cord injury. Curr Pharm Biotechnol 16: 492-505, 2015.

7. Pasquier J, Hoarau-Véchot J, Fakhro K, Rafii A and Abi Khalil C: Epigenetics and Cardiovascular Disease in Diabetes. Curr Diab Rep 15: 108, 2015.

8. Helzner EP and Contrera KJ: Type 2 diabetes and hearing impairment. Curr Diab Rep 16: 3, 2016.

9. Mendez CE, Der Mesropian PJ, Mathew RO and Slawski B: Hyperglycemia and acute kidney injury during the perioperative period. Curr Diab Rep 16: 10, 2016.

10. Usuelli V and La Rocca E: Novel therapeutic approaches for diabetic nephropathy and retinopathy. Pharmacol Res 98: 39-44, 2015.

11. Meng XF, Wang XL, Tian XJ, Yang ZH, Chu GP, Zhang J, Li M, Shi J and Zhang C: Nod-like receptor protein 1 inflammasome mediates neuron injury under high glucose. Mol Neurobiol 49: 673-684, 2014.

12. Dai Y, Zhao X, Chen P, Yu Y, Wang Y and Xie L: Neuropeptide FF promotes recovery of corneal nerve injury associated with hyperglycemia. Inves Ophthalmol Vis Sci 56: 7754-7765, 2015.

13. Wang YH, Chen SY, Wang TD, Hwang BS, Huang TS and Su TC: The relationships among serum glucose, albumin concentrations and carotid atherosclerosis in men with spinal cord injury. Atherosclerosis 206: 528-534, 2009.

14. Readnower RD, Pandya JD, McEwen ML, Pauly JR, Springer JE and Sullivan PG: Post-injury administration of the mitochondrial permeability transition pore inhibitor, NIM811, is neuroprotective and improves cognition after traumatic brain injury in rats. J Neurotrauma 28: 1845-1853, 2011.

15. Liu YX, Zhu KY, Liu YL and Jiang DF: Effects of dietary conjugated linoleic acids on cellular immune response of piglets after cyclosporin A injection. Animal 10: 1660-1665, 2016.
16. El-Gowelli HM, Helmy MW, Ali RM and El-Mas MM: Celecoxib offsets the negative renal influences of cyclosporine via modulation of the TGF- $\beta 1 / \mathrm{IL}-2 / \mathrm{COX}-2 /$ endothelin ET(B) receptor cascade. Toxicol Appl Pharmacol 275: 88-95, 2014.

17. Dao AT, Yamazaki H, Takamatsu H, Sugimori C, Katagiri T, Maruyama H, Zaimoku Y, Maruyama K, Ly TQ, Espinoza L and Nakao S: Cyclosporine restores hematopoietic function by compensating for decreased Tregs in patients with pure red cell aplasia and acquired aplastic anemia. Ann Hematol 95: 771-781, 2016.

18. Working Group For National Survey On Status Of Diagnosis And Treatment Of Childhood Renal Diseases: Multicenter survey of diagnostic and therapeutic status in Chinese childhood patients with steroid-sensitive, relaping/steroid-dependent nephrotic syndrome. Zhonghua Er Ke Za Zhi 52: 194-200, 2014 (In Chinese).

19. Hashimoto A, Kanisawa Y, Fujimi A, Nakajima C, Hayasaka N, Yamada S, Okuda T, Minami S, Yamauchi N, Iwasaki S, et al: Thrombocytopenia and anemia with anti-c-mpl antibodies effectively treated with cyclosporine in a patient with rheumatoid arthritis and chronic renal failure. Intern Med 55: 683-687, 2016.

20. Kumar R, Dogra S, Amarji B, Singh B, Kumar S, Sharma, Vinay K Mahajan R and Katare OP: Efficacy of novel topical liposomal formulation of cyclosporine in mild to moderate stable plaque psoriasis: A randomized clinical trial. JAMA Dermatol 152: 807-815, 2016.

21. Sánchez JA, Alfonso A, Leirós M, Alonso E, Rateb ME, Jaspars M, Houssen WE, Ebel R and Botana LM: Spongionella secondary metabolites regulate store operated calcium entry modulating mitochondrial functioning in SH-SY5Y neuroblastoma cells. Cell Physiol Biochem 37: 779-792, 2015.

22. Dai Y, Sun Q, Zhang X, Hu Y, Zhou M and Shi J: Cyclosporin A ameliorates early brain injury after subarachnoid hemorrhage through inhibition of a Nur77 dependent apoptosis pathway. Brain Res 1556: 67-76, 2014.

23. Yacoub A, Hajec MC, Stanger R, Wan W, Young H and Mathern BE: Neuroprotective effects of perflurocarbon (oxycyte) after contusive spinal cord injury. J Neurotrauma 31: 256-267, 2014.

24. Basso DM, Beattie MS and Bresnahan JC: A sensitive and reliable locomotor rating scale for open field testing in rats. J Neurotraum 12: $1-21,1995$.

25. Han X, Yang N, Xu Y, Zhu J, Chen Z, Liu Z, Dang G and Song C: Simvastatin treatment improves functional recovery after experimental spinal cord injury by upregulating the expression of BDNF and GDNF. Neurosci Lett 487: 255-259, 2011

26. Zhou KL, Zhou YF, Wu K, Tian NF, Wu YS, Wang YL, Chen DH, Zhou B, Wang XY, Xu HZ and Zhang XL: Stimulation of autophagy promotes functional recovery in diabetic rats with spinal cord injury. Sci Re 5: 17130, 2015.

27. Grabher P,Callaghan MF, Ashburner J, Weiskopf N, Thompson AJ, Curt A and Freund P: Tracking sensory system atrophy and outcome prediction in spinal cord injury. Ann Neurol 78: 751-761, 2015.

28. Alexanian AR, Svendsen CN, Crowe MJ and Kurpad SN: Transplantation of human glial-restricted neural precursors into injured spinal cord promotes functional and sensory recovery without causing allodynia. Cytotherapy 13: 61-68, 2011

29. Ni H, Jin W, Yuan B, Zhu T, Wang J, Jiang J, Liang W and Ma Z: Curcumin inhibits the increase of labile zinc and the expression of inflammatory cytokines after traumatic spinal cord injury in rats. J Surg Res 187: 646-652, 2014.

30. Lee SI, Jeong SR, Kang YM, Han DH, Jin BK, Namgung U and Kim BG: Endogenous expression of interleukin-4 regulates macrophage activation and confines cavity formation after traumatic spinal cord injury. J Neurosci Res 88: 2409-2419, 2010.

31. Fenn AM, Hall JC, Gensel JC, Popovich PG and Godbout JP: IL-4 signaling drives a unique arginase ${ }^{+} / \mathrm{IL}-1 \beta^{+}$microglia phenotype and recruits macrophages to the inflammatory CNS: Consequences of age-related deficits in IL-4R $\alpha$ after traumatic spinal cord injury. J Neurosci 34: 8904-8917, 2014.

32. Lau D, Harte SE, Morrow TJ, Wang S, Mata M and Fink DJ: Herpes simplex virus vector-mediated expression of interleukin-10 reduces below-level central neuropathic pain after spinal cord injury. Neurorehabil Neural Repair 26: 889-897, 2012.

33. Lin WP, Lin JH, Cai B, Shi JX, Li WJ, Choudhury GR, Wu SQ, Wu JZ, Wu HP and Ke QF: Effect of adenovirus-mediated RNA interference of IL-1 $\beta$ expression on spinal cord injury in rats. Spinal Cord 54: 778-784, 2016

34. McEwen ML, Sullivan PG, Rabchevsky AG and Springer JE: Targeting mitochondrial function for the treatment of acute spinal cord injury. Neurotherapeutics 8: 168-179, 2011. 
35. White CR, Giordano S and Anantharamaiah GM: High-density lipoprotein, mitochondrial dysfunction and cell survival mechanisms. Chemistry and physics of lipids 199: 161-169, 2016.

36. Fu M, Shi W, Li Z and Liu H: Activation of mPTP-dependent mitochondrial apoptosis pathway by a novel pan HDAC inhibitor resminostat in hepatocellular carcinoma cells. Biochem Biophys Res Commun 477: 527-533, 2016.

37. Scott AJ, Wilkinson AS and Wilkinson JC: Basal metabolic state governs AIF-dependent growth support in pancreatic cancer cells. BMC Cancer 16: 286, 2016.

38. Liu S, Wang X, Lu Y, Xiao J, Liang J, Zhong X and Chen Y: The combined use of cytokine-induced killer cells and cyclosporine a for the treatment of aplastic anemia in a mouse model. J Interferon Cytokine Res 35: 401-410, 2015.
39. Guan N, Ren YL, Liu XY, Zhang Y, Pei P, Zhu SN and Fan Q: Protective role of cyclosporine A and minocycline on mitochondrial disequilibrium-related podocyte injury and proteinuria occurrence induced by adriamycin. Nephrol Dial Transplant 30: 957-969, 2015.

40. Kim SY, Shim MS, Kim KY, Weinreb RN, Wheeler LA and Ju WK: Inhibition of cyclophilin D by cyclosporin A promotes retinal ganglion cell survival by preventing mitochondrial alteration in ischemic injury. Cell Death Dis 5: e1105, 2014.

This work is licensed under a Creative Commons Attribution-NonCommercial-NoDerivatives 4.0 International (CC BY-NC-ND 4.0) License. 